\title{
Deformation and Residual Stress Based Multi-Objective Genetic Algorithm for Welding Sequence Optimization
}

\author{
Jesus Romero-Hdz ${ }^{1}$, Gengis Toledo-Ramirez ${ }^{1}$, Baidya Saha ${ }^{2}$ \\ ${ }^{1}$ Centro de Ingeniería y Desarrollo Industrial, Monterrey, \\ Mexico \\ ${ }^{2}$ Centro de Investigación en Matemáticas, Monterrey, \\ Mexico \\ jaromero@cidesi.edu.mx, gengis.toledo@cidesi.edu.mx, \\ baidya.saha@cimat.com
}

\begin{abstract}
Compared to deformation, residual stress has not been taken into account in the literature when it comes to welding process optimization. It also plays an important role to measure the weld quality. This paper reports the implementation of a multi-objective based Genetic Algorithm (GA) for welding sequence optimization, in which both structural deformation and residual stress are offered equal importance. The optimal weights between them are dynamically selected through optimizing a multi-objective fitness function in an iterative manner. A thermo-mechanical finite element analysis (FEA) was used to predict both deformation and residual stress. We chose the elitism selection approach to ensure that the three best individuals are copied over once into the next generation to facilitate convergence by preserving good candidates which can offer an optimal solution. We exploited a sequential string searching algorithm into single point crossover method to avoid the repetition of single beads into the sequence. We utilized a bit string mutation operator by changing the direction of the welding from one bead chosen randomly from the sequence. Welding simulation experiments were conducted on a typical widely used mounting bracket which has eight seams. Multi-objective based GA effectively reduces the computational complexity over exhaustive search with significant reduction of both structural deformation $(\sim 80 \%)$ and residual stress $(\sim 15 \%)$.
\end{abstract}

Keywords: Multi-objective optimization, genetic algorithm, residual stress.

\section{Introduction}

Fusion welding processes still very common in manufactutring, because they provide several advanteges in terms of cost, flexibility and design reliability. Gas Metal Arc Welding (GMAW) joins metals by simultaneously melting the base metal and adding a filler material to the joint to form a pool of molten material (the weld pool). Then, it cools down to form a joint that can be same or stronger than the base metal [9]. Welding is extensively used in a wide range of industries such as automotive, shipbuilding, 
aerospace, construction, pipelines, nuclear, pressure vessels, heavy and earth-moving equipment $[22,13]$.

The scope of this research is limited to GMAW process. Welding deformation and effective residual stress derive several negative impacts to the manufacturing process, adding additional cost in various ways, such as constraints in the design phase, extra operations, cost of quality and overall capital expenditure. Welding deformation and residual stress can be minimized by finding a suitable welding sequence. The industrial practice of welding sequence optimization is to select the best sequence by experience and sometimes running a simplified design of experiments which typically does not guarantee the optimal sequence [19]. Since conducting many real welding experiments is very expensive, welding deformation and effective residual stress are computed through a welding simulation software based on Finite Element Methods (FEM). Thermo-mechanical models can work under various welding conditions and geometric configurations. However, it is computationally very expensive and time consuming.

The best welding sequence can be achieved through a full factorial design procedure. For full factorial design, the total number of welding configurations can be computed by $N=n^{r} \times r$ !, where $n$ and $r$ are the number of welding directions and beads (seams or segments) respectively. This number grows exponentially with the number of welding beads. For example, a complex weldment like an aero-engine assembly, it might have 52-64 weld segments [14]. Hence, the full factorial design is not feasible for industrial applications.

In this research, we implemented an iterative dynamic weight selection based multiobjective GA for welding sequence optimization. We make the following technical contributions in this paper:

- Multi-objective based GA effectively reduces the computational complexity over extensive search. In this research we have used eight weld seams and two welding directions. The number of welding configurations for exhaustive search is 10,321,920. In this experiment we achieved the optimal solution through GA after executing the welding simulation for 42 welding configurations. This is the minimum number of configurations necessary to find the optimal solution which was found based on the general Markov chain model of GA. The average execution time for each welding configuration using thermo-mechanical FEM approach is 30 minutes. Thus we saved significant amount of computational time.

- Literature reveals that both deformation and effective residual stress [6] measure the weld quality. Though, deformation was frequently used in the past studies, however, effective stress has been ignored as demonstrated in the Table 1. This paper combines both structural deformation and residual stress as a measure of weld quality and offers equal importance to both of them. The optimal weight was chosen through dynamic selection of weights in an iterative manner while optimizing the welding sequence optimization through multi-objective GA. We exploited a fitness function by the weighted linear combination of the inverse of the maximum structural deformation and effective residual stress. 
- We expedited the convergence of the GA through the elitism selection approach and we copied the three best individuals into the next generation and preserved the best individuals which might lead to optimal solution.

- We tailored the single point crossover algorithm for the welding sequence optimization to avoid the repetition of single beads in the welding sequence by incorporating a sequential string searching algorithm into the single point crossover method.

- We implemented the bit string mutation algorithm by changing only the direction of the welding on one bead selected randomly from the sequence obtained by crossover algorithm instead of changing the bead itself to avoid the repetition of single bead in the welding sequence.

Experiments were conducted through the well-known simulation software Simufact Welding $($ on a mounting bracket, which is widely used in telescopic jib [5] and automotive industries [26,12]. Experimental results demonstrate that best welding sequence can reduce significant amount of structural deformation $(\sim 80 \%)$, effective residual stress $(\sim 15 \%)$ over the worst sequence.

The organization of the paper is as follows. Section 2 presents literature review. Section 3 discusses the thermal and mechanical analysis of FEM. Proposed dynamic weight selection based multi-objective GA for welding sequence optimization and its convergence analysis are presented in section 4 . Results and discussions are demonstrated in section 5. Section 6 concludes this work. Relevant references are listed at the end of the paper.

\section{Literature Review}

Several authors have implemented GA for welding sequence optimization. A brief review is presented in Table 1. Furthermore, Table 2 shows a review of their validation methods.

Table 1. Literature review on implemented GA fitness function.

\begin{tabular}{|c|c|c|c|c|c|}
\hline \multirow[b]{2}{*}{ Author } & \multicolumn{5}{|c|}{ Main functions } \\
\hline & $\begin{array}{l}\text { Trajectory } \\
\text { time }\end{array}$ & Deformation & $\begin{array}{l}\text { Residual } \\
\text { stress }\end{array}$ & Temperature & Others \\
\hline [27] & Yes & Yes & No & No & No \\
\hline [17] & Yes & No & No & Yes & Robot joint movements \\
\hline [20] & No & Yes & No & No & No \\
\hline [23] & No & Yes & No & No & No \\
\hline [4] & No & Yes & No & No & $\begin{array}{l}\text { Stiffness and stress } \\
\text { constraints }\end{array}$ \\
\hline [13] & No & Yes & No & Yes & No \\
\hline [15] & No & Yes & No & No & No \\
\hline
\end{tabular}

Among the studies mentioned in Table 1, Xie et al. [27] and Kim et al. [17] proposed multi-objective GA that are discussed below. Xie and Hsieh [27] have implemented 
Jesus Romero-Hdz, Gengis Toledo-Ramirez, Baidya Saha

Table 2. Literature review on GA validation methods.

\begin{tabular}{llllll}
\hline \multirow{2}{*}{ Author } & \multicolumn{5}{c}{ Validation type } \\
\cline { 2 - 6 } & $\begin{array}{l}\text { Specimen } \\
\text { FEA }\end{array}$ & $\begin{array}{l}\text { Specimen } \\
\text { tryout }\end{array}$ & $\begin{array}{l}\text { Real } \\
\text { part Real part try- Others } \\
\text { out }\end{array}$ \\
\hline$[27]$ & No & No & Yes & No & No \\
{$[17]$} & No & No & No & No & Virtual trajectory Sim \\
{$[20]$} & No & No & Yes & No & No \\
{$[23]$} & Yes & No & No & No & No \\
{$[4]$} & No & No & Yes & No & No \\
{$[13]$} & Yes & Yes & Yes & No & No \\
{$[15]$} & Yes & No & No & No & No \\
\hline
\end{tabular}

GA for finding a combined clamping and welding sequence. A multi-objective fitness function is taken into account to minimize cycle time (gun travel path) and assembly deformation as shown in Equation 1. FEM was used to evaluate the fitness function on automotive parts by spot welding process.

$$
\begin{array}{r}
\operatorname{Min} F=w_{1} \frac{D_{i}}{D_{0 i}}+w_{2} \frac{C}{C_{0}}, \\
i=1,2,3 \ldots N,
\end{array}
$$

where, $w_{1}$ and $w_{2}$ are weights that define the importance of each sub-function; $D_{i}$ is the total deformation on every single node for the actual generation. $D_{0 i}$ is the total deformation on every single node for the initial generation; $C$ is the cycle time for the actual generation and $C_{0}$ is the cycle time for the initial generation. Notice that $\frac{D_{i}}{D_{0 i}}$ and $\frac{C}{C_{0}}$ are considered as normalized functions because the units of deformation and cycle time are different.

Kim et al. [17] have implemented GA using a multi-criteria fitness function (Equation 2). This function includes the minimization of gun travel time, avoidance of thermal distortion and smooth robot joint movement. The criteria considered here are Euclidian distance between weld seams, a $30 \mathrm{~mm}$ distance considered as heat affected zone and total change of the robot joints respectively. This algorithm is suitable for different arc welding operations such as multi weld lines: singlepass or multipass:

$$
\operatorname{Min} F=\operatorname{Min}\left(w_{1} g_{1}+w_{2} g_{2}\right),
$$

where: $w_{1}$ and $w_{2}$ are weights. The sub-function that involves gun travel time and distortion criteria $g_{1}$ is defined by

$$
g_{1}=\sum_{a_{i j} \in T} x_{i j}
$$

where: $T$ is a trajectory,

$$
x_{i j}=\left\{\begin{array}{c}
c_{i j} \text { if } a_{i j} \notin h_{i j} \\
c_{i j}+M_{1} \text { if } a_{i j} \in h_{i j}
\end{array}\right\},
$$




$$
c_{i j}=\left\{\begin{array}{c}
l_{i j} \text { if } a_{i j} \in W \\
l_{i j}+M_{2} \text { if } a_{i j} \notin W
\end{array}\right\},
$$

where: $h_{i j}$ is the heat affected zone for each weld seam $a_{i j}$ in $W ; l_{i j}$ is the arc length $a_{i j} ; A$ is a set of $\operatorname{arcs} a_{i j}$ from each node $i \in N$ to each node $j \in N ; N$ is a finite set of nodes in the seam $w$. $W$ is a set of arcs that represents a weld seam $W \subseteq A$. For the sub-function that involves the smooth robot joint movements $g_{2}$ is defined by:

$$
g_{2}=\sum_{a_{i j} \in T} \sum_{k \in J} \theta_{i j k}
$$

where: $\theta_{i j k}$ is the angle of change for a joint $k$ from one node $i$ to other node $j$ from the set $a_{i j} . J$ is a set of robot joints. The penalty terms $M_{1}$ and $M_{2}$ are sufficiently large numbers. $M_{1}$ ensures that only seams out of the heat affected zone criteria $(30 \mathrm{~mm})$ will be selected. $M_{2}$ ensures that only valid segments are selected and all of them will be traveled.

From Table 1, it was shown that past studies have considered deformation and some of them exploited trajectory time of the robot, temperature, robot joint movements, stiffness and stress constraints as the measure of the weld quality. However, residual stress, which is also an important measure for the weld quality [6] has been ignored in the GA studies utilized for weld sequence optimization. In this research we exploited both deformation and effective residual stress as the measure of the weld quality and exploited both of them equally in the fitness function using iterative dynamic weight selection based multi-objective GA that are discussed in the subsequent sections.

\section{Welding Simulation Framework}

In order to present our approach we overview the welding simulation framework. This is important because the fitness function is computed using FEA.

\subsection{Thermal Analysis}

Weld process modeling (WPM) is a very complex task. The physics of heat generation has as a fundamental principle the law of conservation of energy. The heat equation can be written in the following form [9] (Conservation of Energy or Heat Equation):

$$
\begin{aligned}
\dot{h} & =\nabla \cdot q+Q, \\
q & =-\kappa \nabla T, \\
d h & =\rho c_{p} d T,
\end{aligned}
$$

where $h, q, Q, T$ and $\nabla T$ represent enthalpy, thermal flux, power density function, temperature and temperature gradient respectively. $\kappa$ and $c_{p}$ represent thermal conductivity tensor and specific heat respectively and both are temperature dependent material properties. Temperature history in every node is computed by the transient heat conduction equation 7a, where the change in enthalpy $\dot{h}=\rho C_{p} \frac{\partial T}{\partial t}$, where: $\rho$ is the material density 
$\left(\mathrm{g} / \mathrm{mm}^{3}\right), C_{p}$ specific heat capacity $\left(\mathrm{J} /\left(\mathrm{g}^{\circ} \mathrm{C}\right)\right), T$ is the current temperature $\left({ }^{\circ} \mathrm{C}\right), q$ flux heat vector $\left(W / \mathrm{mm}^{2}\right), Q$ is the internal heat generation rate $\left(W / \mathrm{mm}^{3}\right), t$ is the time $(s), \nabla$ is the spatial gradient operator $\mathrm{x}, \mathrm{y}, \mathrm{z}\left(\frac{\partial}{\partial x}, \frac{\partial}{\partial y}, \frac{\partial}{\partial z}\right)$. The heat flux is the amount of energy that flows through a particular surface per unit area per unit time. This is defined by the differential form of Fourier's Law of thermal conduction equation described in $7 \mathrm{~b}$. Here $\kappa$ is the temperature-dependent thermal conductivity matrix $\left(\mathrm{J} / \mathrm{mms}^{\circ} \mathrm{C}\right)$ and $\nabla T$ is the temperature gradient.

Typically, the complexity of the heat generation physics in the weld puddle is simplified by using a heat input model or well known as welding simulation models. The classical approach in Computational Welding Mechanics (CWM) is to ignore fluid flow and use a heat input model where heat distribution is prescribed. The given heat input replaces the details of the heat generation process and focus on larger scales. Moreover, the modeling of fluid flow and pertaining convective heat transfer may be integrated with a CWM model.

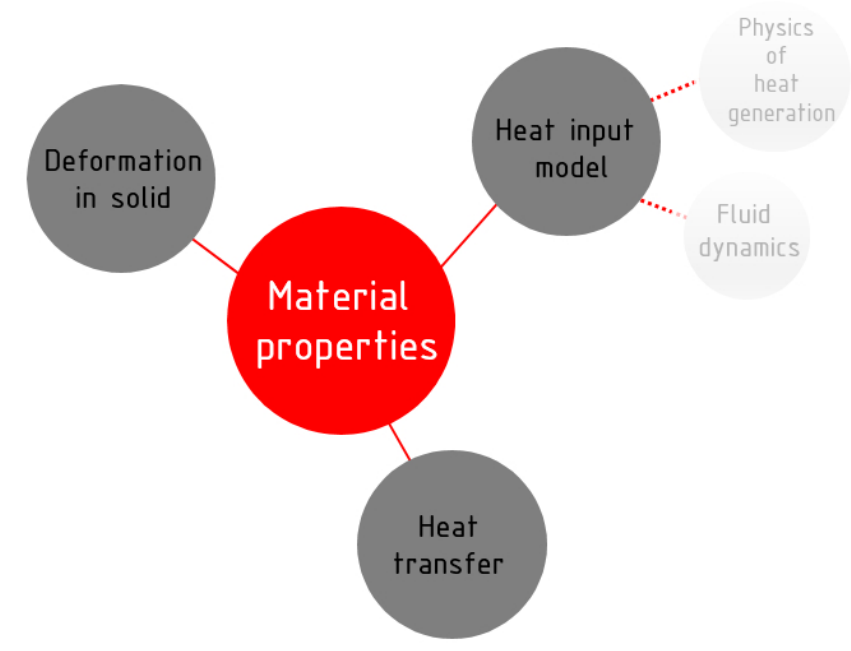

Fig. 1. Welding simulation.

The most common used model for fusion welding processes is the well-known Goldak double ellipsoidal heat distribution. This heat input model combines two ellipsoidal heat sources to achieve the expected steeper temperature gradient in front of the heat source and a less steep gradient at the trailing edge of molten pool. This two heat sources are defined by Front heat distribution:

$$
Q\left(x^{\prime}, y^{\prime}, z^{\prime}, t\right)=\frac{6 \sqrt{3} f_{f} Q_{w}}{\pi \sqrt{\pi} a b c_{f}} e^{\left(\frac{-3 x^{2}}{a^{2}}\right)} e^{\left(\frac{-3 y^{2}}{b^{2}}\right)} e^{\left(\frac{-3 z^{\prime 2}}{C_{f}^{2}}\right)}
$$


Rear heat distribution:

$$
Q\left(x^{\prime}, y^{\prime}, z^{\prime}, t\right)=\frac{6 \sqrt{3} f_{r} Q_{w}}{\pi \sqrt{\pi} a b c_{r}} e^{\left(\frac{-3 x^{2}}{a^{2}}\right)} e^{\left(\frac{-3 y^{\prime 2}}{b^{2}}\right)} e^{\left(\frac{-3 z^{2}}{C_{r}{ }^{2}}\right)},
$$

where: $f_{f}$ is the fraction factor of heat deposited in the front part, $f_{r}$ is the fraction factor of heat deposited in the rear part. Those factors must satisfy the relation $f_{f}+f_{r}=2$. $a$ is the width, $b$ is depth, $c_{r}$ is the length of the rear ellipsoid $\mathrm{y} c_{f}$ is the length of the front ellipsoid.

These parameters are physically related to the shape of the weld puddle Figure 2. Width and depth are commonly taken from the cross section, the authors recommend to use a half of parameter $a$ for the front fraction and two times $a$ for the rear fraction. For a linear trajectory along axis $z$, is defined by $z^{\prime}$ :

$$
z^{\prime}=z+v(\tau-t),
$$

where $z$ actual coordinate $z, v$ is travel speed, $\tau$ is a delay factor and $t$ is the time. The heat available from the heat source is defined by:

$$
Q_{w}=\eta I E,
$$

where $\eta$ heat source efficiency, $I$ is the current $(A), E$ is the voltage $(V)$.

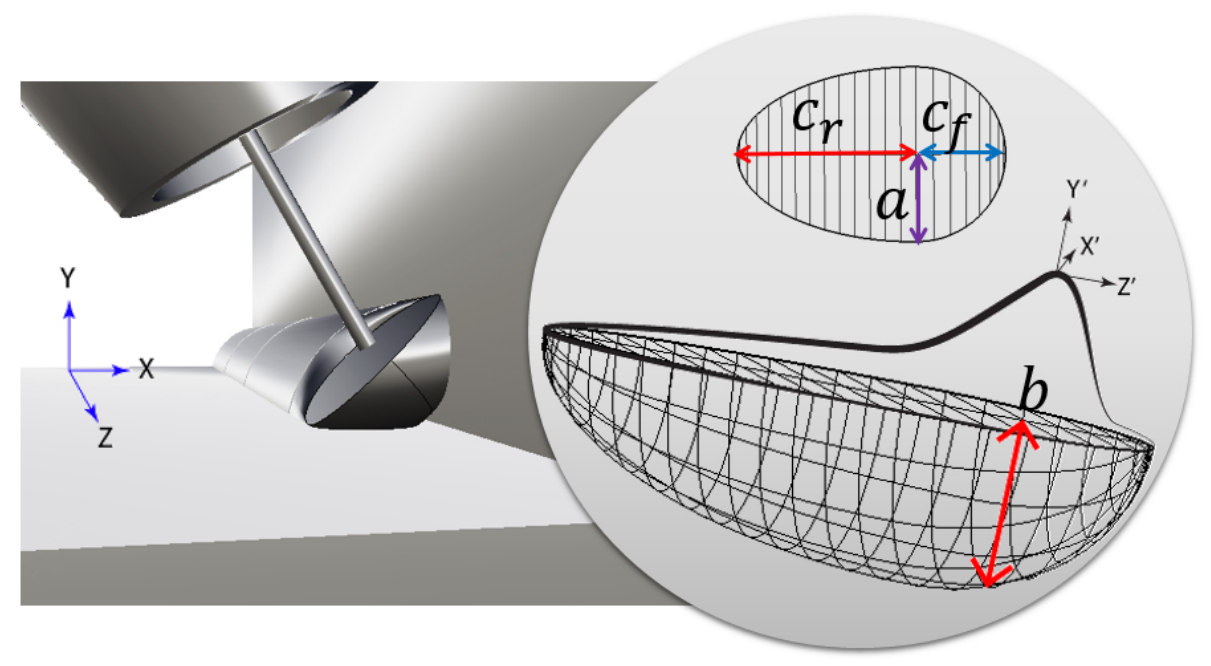

Fig. 2. Goldak double ellipsoidal model.

Thus the heat input model in CWM must be calibrated with respect to experiments or obtained from WPM models. Therefore, the classical CWM models have some limitations in their predictive power when used to solve different engineering problems. For example, they cannot prescribe what penetration a given welding procedure will 
give. The appropriate procedure to determine the heat input model is therefore important in CWM [21].

The FEM software solves this time dependent system of partial differential equations on a domain defined by a FEM mesh. The domain is dynamic in that it changes with each time step as filler metal is added to the weld pass. The initial condition is often assumed to be the ambient temperature but the domain can be initialized to any initial temperature field. The heating effect of the arc is often modeled by a double ellipsoid power density distribution that approximates the weld pool as measured from macrographs of the cross-section of several weld passes. A convection boundary condition $q=h\left(T-T_{a m b}\right)$ with convection coefficient $h$ and ambient temperature $T_{m}$ usually is applied to external surfaces. The FEM formulation of the heat equation leads to a set of ordinary differential equations that are integrated in time using a backward Euler integration scheme.

\subsection{Mechanical Analysis}

The temperature history from the thermal analysis was used as a series of loads in the structural analysis. In this phase, the temperature history from the thermal cycle of each node is taken as an input and it is used as a node load with temperature dependent material properties. The solid model mesh used for the mechanical analysis was also used for the thermal analysis where each increment of weld deposition corresponded to one load step. Because phase transformation has an insignificant effect on the welding residual stress and distortion, the total strain $\varepsilon^{\text {total }}$ (assuming negligible contribution from solid state phase transformation) can be decomposed into three components as follows: $\varepsilon^{\text {total }}=\varepsilon^{e}+\varepsilon^{p}+\varepsilon^{\text {th }}$, where $\varepsilon^{e}, \varepsilon^{p}$, and $\varepsilon^{\text {th }}$ represent elastic, plastic and thermal strain respectively. In the welding process, changes in stress caused by deformation are assumed to travel slowly compared to the speed of sound. So, at any instant, an observed group of material particles is approximately in static equilibrium, i.e., inertial forces are neglected.

In rate independent plasticity, viscosity is zero and viscous forces are zero. In either the Lagrangian or the Eulerian reference frame, the partial differential equation of equilibrium is, at any moment is given by the conservation of momentum equation that is mentioned below [8] (Conservation of Momentum Equation):

$$
\begin{aligned}
\nabla . \sigma+f & =0, \\
\sigma & =D \varepsilon \\
\varepsilon & =\left(\nabla u+(\nabla u)^{T}+(\nabla u)^{T} \nabla u\right) / 2,
\end{aligned}
$$

where $\nabla, \sigma, f, D, \varepsilon$ and $u$ represent partial differential, cauchy strss, total body force, temperature dependent material property (elastic matrix relevant to the modulus of elasticity and Poisson's ratio), the Green-Lagrange strain and displacement vector respectively. $\nabla u$ represents the displacement gradient.

The mechanical model is based on the solution of three partial differential equations of force equilibrium illustrated in Equation 12. In the FEM formulation, Equation 12 is transformed and integrated over the physical domain, or a reference domain with a 
unique mapping to the physical domain [9]. The simulation software solves this partial differential equation for a viscothermo-elasto-plastic stress-strain relationship. The initial state often is assumed to be stress free. Dirichlet boundary conditions constrain the rigid body modes. The system is solved using a time marching scheme with time step lengths of approximately 0.1 second during welding and 5 second during cooling phase.

\section{Proposed Genetic Algorithm Based Welding Sequence Optimization framework}

Genetic Algorithms emulate natural selection of a set of individuals in order to search the best solution to a problem [10]. The genetic configuration of each individual is a possible solution. GA starts with an initial population and those are submitted to an evolutionary process in such way that the best adapted individuals will continue to reproduce among them and over several generations the best adapted stands out. We tailor the GA for the welding sequence optimization: selection, cross-over, and mutation to avoid the repetition of single bead that is discussed below.

\subsection{String Representation of Welding Sequence}

Being $Q$ the welding application and $S$ a set of all possible sequences of $Q$, each sequence $s \in S$ represents a possible sequence which minimizes the overall structure deformation and residual stress. Each sequence has $N$ weld seams, here called genes $s=\left\{x^{1}, x^{2}, x^{3}, \ldots x^{N}\right\}$, these are a combination of real numbers $\forall n=1,2,3, \ldots N$. In this approach every seam can be welded in two directions and it is represented by a positive sign if $\circlearrowleft$ or $\uparrow$ or $\leftarrow$ or negative sign if $\circlearrowright$ or $\downarrow$ or $\rightarrow$.

\subsection{Initialization of welding sequence}

The algorithm starts with an initial population $P=\left\{s_{j}\right\}$, where elements of the set of sequences are called "individuals" $j=1,2,3, \ldots J$. Their genes are generated randomly and special considerations taken in order to avoid repeated seam in the same welding sequence.

\subsection{Deformation Based Fitness Value}

Within the scope of natural selection, the individual eligibility is regarded as the degree of adaptability. In this paper we have implemented a multi-objective fitness function that takes into account deformation and residual stress and returns a real number (weighted linear combination of maximum deformation and residual stress of the structure) $f\left(s_{j=1}^{J}\right) \Rightarrow \mathbb{R}$ that measures the adaptability of each sequence:

$$
F\left(s_{j}\right)=\sum_{i=1}^{I} w_{i} f_{i},
$$


where $w_{i}$ is the weight that defines the importance of each sub-function. These weights are computed dynamically in each iteration $t$ with the equation 14 [7]:

$$
w_{i}(t)=\frac{\sum_{\substack{j=1 \\ j \neq i}}^{I}\left|f_{j}\left(x_{t-1}\right)\right|}{(I-1) \times \sum_{j=1}^{I}\left|f_{j}\left(x_{t-1}\right)\right|},
$$

where $I$ is the number of sub-functions, $i=1 \ldots I, x_{t-1}$ is the best individual among solutions of the population in the previous generation $P_{t-1} . w_{i}(t)$ is the dynamic weight satisfying the following conditions,

$$
0 \leq w_{i}(t) \leq 1 \text { and } \sum_{i=1}^{I} w_{i}(t)=1
$$

where $t$ represents the iteration step of the GA algorithm. $f_{1}$ is a sub-function that takes into account the final deformation on the structure and it is computed by FEA. Final deformation is defined by the equation 18 :

$$
f\left(s_{j}\right)=1 /\left(\operatorname{Max}\left(D_{i}\right)+\varepsilon\right),
$$

where $D_{i}$ is the total deformation on every node defined by

$$
\begin{gathered}
D_{i}=\sqrt{{d_{x_{i}}{ }^{2}+d_{y_{i}}{ }^{2}+d_{z_{i}}{ }^{2}},} \\
i=1,2,3 \ldots N
\end{gathered}
$$

$d_{x_{i}}, d_{y_{i}}$, and $d_{z_{i}}$ are the deformation of node $i$ along $x, y$, and $z$ axis respectively. $\varepsilon$ is a very small number which was used to offer continuity to the fitness function when the value of the maximum deformation is zero.

$f_{2}$ is a sub-function that takes into account effective stress. It is also computed by FEA and it is defined by the following equation .

$$
\begin{gathered}
f\left(s_{j}\right)=1 /\left(\operatorname{Max}\left(E_{i}\right)+\varepsilon\right), \\
E_{i}=\frac{\sqrt{\left(\sigma_{1 i}-\sigma_{2 i}\right)^{2}+\left(\sigma_{2 i}-\sigma_{3 i}\right)^{2}+\left(\sigma_{3 i}-\sigma_{1 i}\right)^{2}}}{\sqrt{2}}, \\
i=1,2,3 \ldots N
\end{gathered}
$$

where $\sigma_{1}, \sigma_{2}, \sigma_{3}$ are the maximum, intermediate and minimum principal stresses. $\varepsilon$ is a very small number which was used to offer continuity to the fitness function when the value of the maximum effective stress is zero. 


\subsection{Welding Sequence Selection Algorithm}

Selection is an important sub-routine where individuals are chosen from the actual population for later procreation. Good selection algorithm expedites the convergence of the welding sequence. As a selection procedure, we first implemented a truncation procedure where the population is sorted by ascending fitness values, then a proportion $\mu$ of the individuals are taken based on fitness value. The proportion $\mu$ is computed by the fraction of the individual fitness value to the sum of the fitness values of all the samples as shown in Fig. 3.

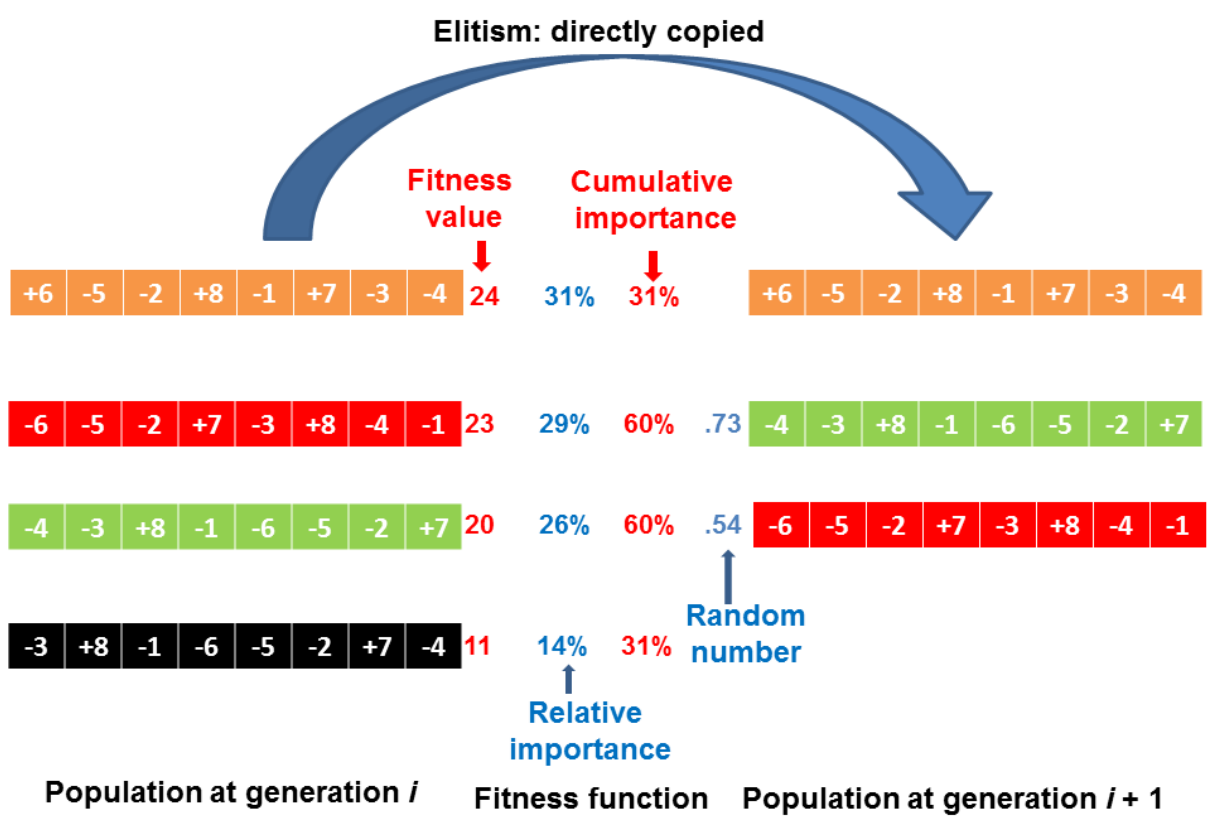

Fig. 3. Selection procedure with elitism function.

\subsection{Crossover for Generating New Welding Sequences}

Crossover is analogous to reproduction, new individuals are created from the selected parents. Each couple of selected individuals $s_{1}$ and $s_{2}$ exchange their genes and make two new individuals, $s_{1}^{\prime}=s_{1} \times s_{2}$ and $s_{2}^{\prime}=s_{2} \times s_{1}$. Several methods for crossover are reported in literature such as arithmetic, heuristic, single or multi-point, uniform, cycle, partially mapped and order [16,11]. In this paper we implemented a single point crossover as demonstrated in Fig. 4 where a random number defines the cut point $a \in[1, N]$. Later, the descendants are defined by equations 20 y 21 respectively. 


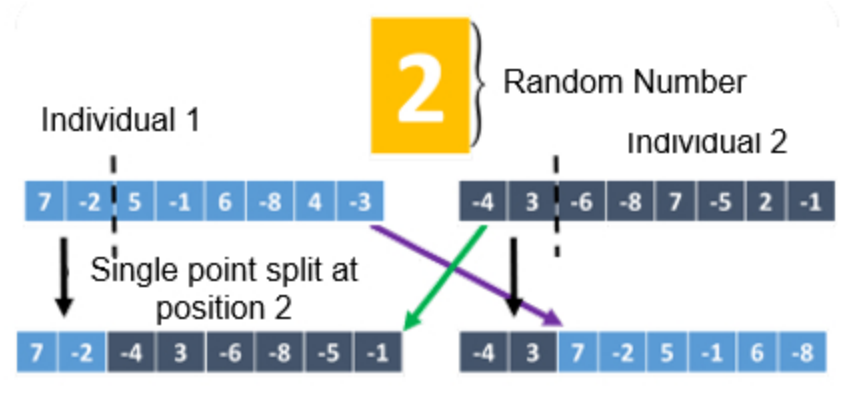

New individual 1

New individual 2

Fig. 4. Single point crossover operator.

To avoid the repetition of the weld seam in the same welding sequence during crossover we implement a repeated string validation algorithm, the pseudo-code of which is illustrated below.

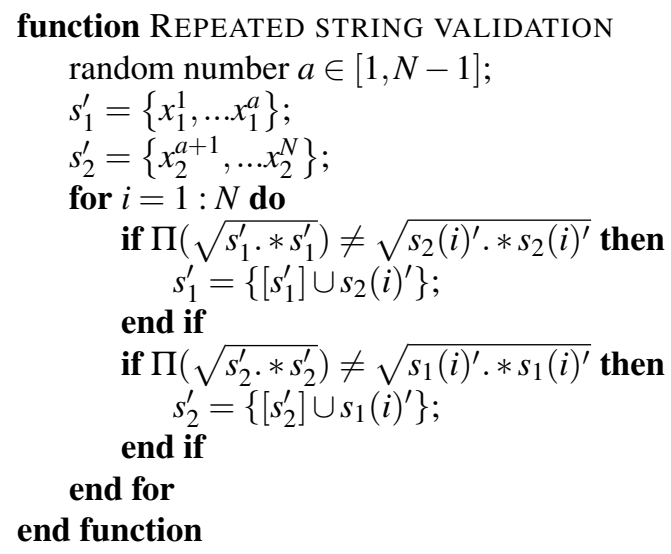

$$
\begin{aligned}
& s_{1}^{\prime}=\left\{\left[x_{1}^{1}, \ldots x_{1}^{a}\right],\left[x_{2}^{a+1}, \ldots x_{2}^{N}\right]\right\}, \\
& s_{2}^{\prime}=\left\{\left[x_{2}^{1}, \ldots x_{2}^{a}\right],\left[x_{1}^{a+1}, \ldots x_{1}^{N}\right]\right\} .
\end{aligned}
$$

\subsection{Mutation for Generating New Welding Sequences}

Mutation alters one or more individual genes from its actual configuration. It occurs during evolution in a low incidence according to a defined mutation probability. Some of the operators found in literature are bit string, delta, invert and swap [24,1,18]. Here we have used a bit string operator in order to change the direction of welding only rather than the welding seam itself as shown in Fig. 5 to avoid the repetition of the weld seam. 


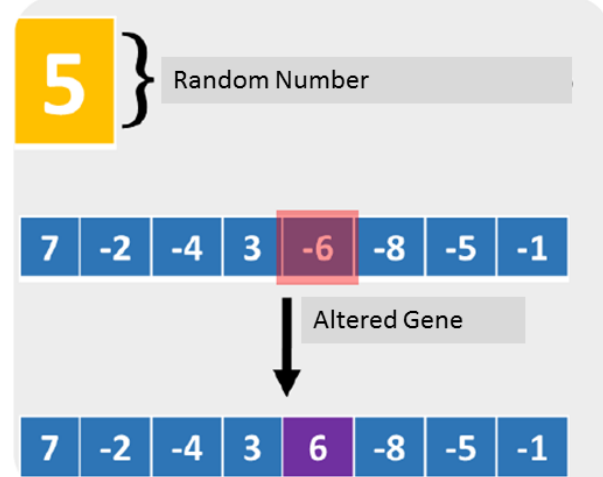

Fig. 5. Bit string mutation operator.

\subsection{Elitism Based Welding Sequence Selection Algorithm}

The Elitism function is a practical variant that ensures that the best individual in the actual population $s_{\text {best }} \in P_{t}$ and current generation $t$ to carry over to the next generation $P_{t+1}$ as shown in Fig. 3. Elitist based selection algorithm guarantees that the convergence obtained by the GA will follow monotone decreasing behaviour over generations, $\left[s_{\text {best }} \in P_{t}\right] \rightarrow P_{t+1}$.

\subsection{Pseudo-code and Flowchart of the Proposed Iterative Genetic Algorithm for the Welding Sequence Optimization}

The following algorithm is a repetitive process where the population is going to be changing over the generations $P_{t}=\left(s_{1}(t), s_{2}(t), \ldots s_{J}(t)\right) \in S$. The pseudo-code for the proposed GA based welding sequence optimization is given below.

function $\mathrm{GA}($ Min $D: Q)$

Input: $P_{0}=\left(s_{1}(t), s_{2}(t), \ldots, s_{J}(t)\right) \in S$

Output: $s_{\text {best }}$, the best sequence that shows the minimum value of the weighted linear combination of deformation and residual stress.

$t \leftarrow 0$

Initialize $P_{t} \in S$;

We assign arbitrary positive real numbers to $w_{i}(0), i=1, \ldots, I$, satisfying the conditions mentioned in equation 15 .

while !terminating condition do

$$
t++ \text {; }
$$

Compute the fitness function $F\left(s_{j}\right)=\sum_{i=1}^{I} w_{i}(t-1) f_{i}(t-1) ; j=1,2, \ldots, J$ $\forall s_{j} \in P_{t-1}$

Select $P_{t}$ from $P_{t-1}$ based on the relative importance of the value of the individual fitness function $F\left(s_{j}\right) . ; / *$ Priority given to the welding sequences based on weighted linear function of less deformation and residual stress */ 
Crossover $P_{t} \leftarrow P_{t}$; /* String searching based single point crossover $* /$ Mutation $P_{t} \leftarrow P_{t}$; /* Change the direction of the welding of one seam */ Evaluate $F\left(s_{j}\right), j=1,2, \ldots, J$;

elitism $P_{t} \leftarrow s_{\text {best }}$ from $P_{t}$; /* Elitism based selection approach */

Compute $f_{i}\left(s_{\text {best }}\right), i=1,, 2, . ., I$.

$$
\sum_{j=1}^{I}\left|f_{j}\left(s_{\text {best }}\right)\right|
$$

If $\sum_{i=1}^{I}\left|f_{i}\left(s_{\text {best }}\right)\right| \neq 0$ then compute, $w_{i}(t)=\frac{j \neq i}{(I-1) \times \sum_{j=1}^{I}\left|f_{j}\left(s_{\text {best }}\right)\right|}, i=1,2, \ldots, I ;$

\section{end while}

return $s_{\text {best }}$ from $P_{t}$.

\section{end function}

Fig. 6 describes the flowchart of the GA based welding sequence optimization approach.

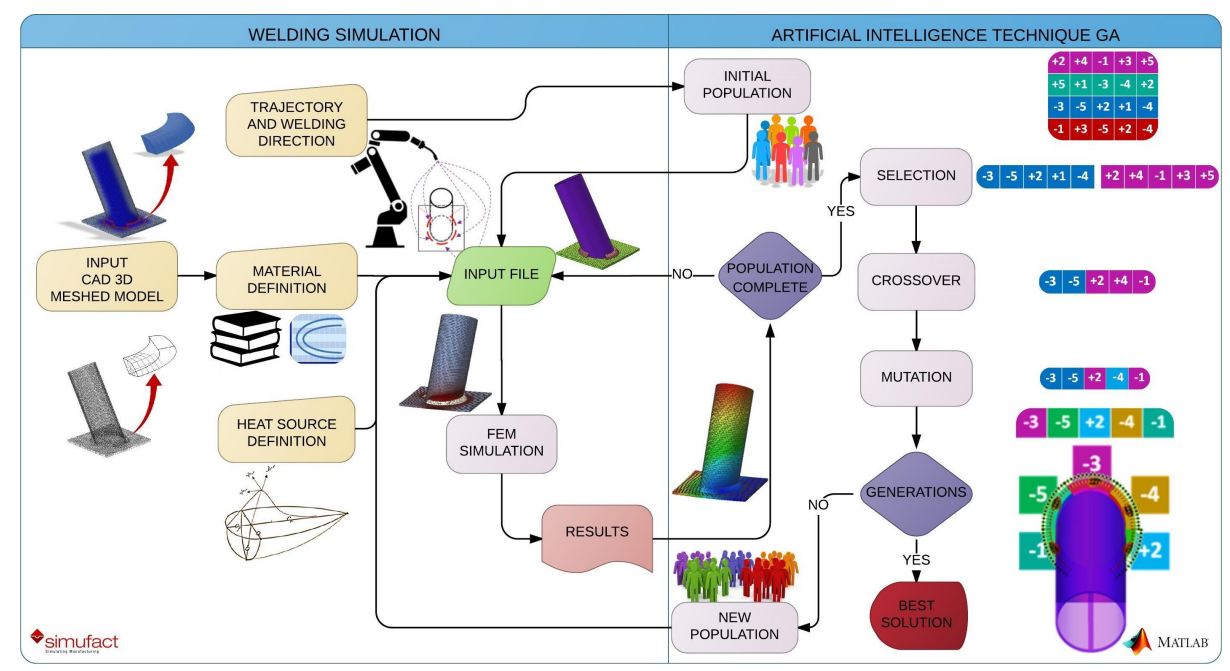

Fig. 6. GA based welding sequence optimization approach.

\subsection{Convergence Analysis of the Genetic Algorithm}

Aytug and Koehler [2,3] showed that for a general Markov chain model of genetic algorithm with elitism, an upper bound for the number of iterations $t$ required to generate a population $S^{+}$which consists entirely of minimal solutions has been generated with probability $\alpha \in(0,1)$, is given by:

$$
t \geq\left\lceil\frac{\ln (1-\alpha)}{n \ln \left(1-\min \left\{\mu^{l},(1-\mu)^{l}\right\}\right)}\right\rceil,
$$


where, $l$ is the length of the chains that represent the individual, $n$ is the population size and $\mu \in(0,1)$ is the mutation rate. $\lceil x\rceil$ is the smallest integer greater than or equal to $x$. Studniarski [25] showed that for multi-objective optimization, the (possibly unknown) number $m$ of these solutions is bounded from below by some known positive integer $\bar{m}$. Suppose also that there exists a number $\beta \in(0,1 / \bar{m})$, an upper bound for the number of iterations $t$ is given by:

$$
t \geq\left\lceil\frac{\ln (1-\alpha)}{\ln \left(1-(\bar{m} \beta)^{l}\right)}\right\rceil .
$$

If no non-trivial lower bound $\bar{m}$ is known, we may always use $\bar{m}=1$.

\section{Experimental Results}

This section consist of the following subsections, fist we describe the study case. Second, we introduce the parameters we have used in terms of GA configuration. Third, convergence analysis of multi-objective GA is given and last, we present the effects of welding sequence optimization over the quality variables we targeted before.

\subsection{Study Case}

We chose a study case of welding a mounting bracket shown in Fig. 7 and 8 which is typically used in telescopic jib [5], automotive industries [26,12]. We conducted

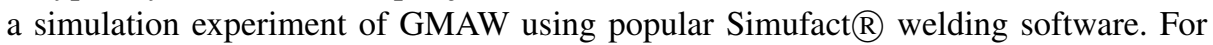
details about the software, please see [13]. We implemented a multi-objective GA algorithm for choosing the best welding sequence having minimum weighted linear combination of structural deformation and effective residual stress and we demonstrated the effects of welding sequence on the weld quality (structural deformation and effective stress) by analyzing the structural deformation and residual stress caused by welding of the four sequences (best, second best, worst and second worst found by GA). Fig. 7 shows geometries of different mounting brackets that can be found frequently in heavy equipment, vehicles, ships, and Fig. 8 illustrates the engineering drawing with all specifications of the mounting bracket used in this experiment.
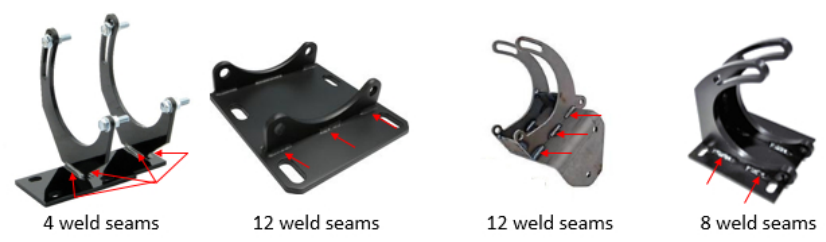

Fig. 7. Different mounting brackets in the market. 


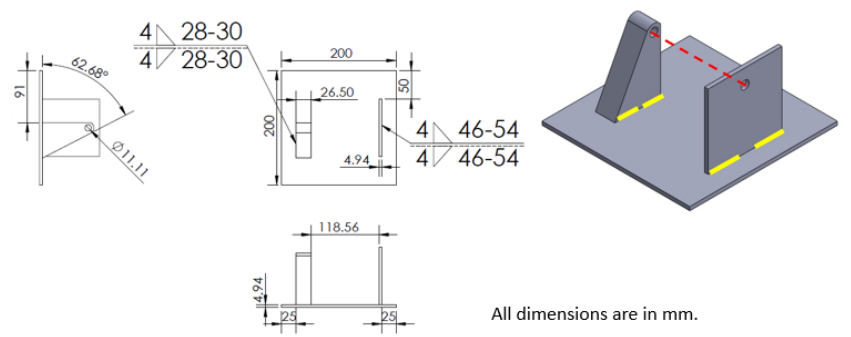

Fig. 8. Study case with 8 seams.

\subsection{Parameters Used for this Study}

Table 3 shows the GA parameters used in the simulation experiment. We considered 7 generations to converge the GA, initial population size as 6 , crossover probability as $50 \%$. We copy three best candidates of the current generation to the next generation using elitism based selection mechanism. We implemented single point cross-over method for new sample reproduction. We also implemented single bit string mutation operator and changed the welding direction of a randomly selected welding seam instead of welding seam itself to avoid the repetition of the welding seam in the sequence.

Table 3. GA parameters.

\begin{tabular}{lc}
\hline Parameter & Value \\
\hline Initial population size & 6 \\
\hline Generations & 7 \\
\hline Elitism candidates & 3 \\
\hline Crossover $\%$ & $50 \%$ \\
\hline Mutation operator & bit string \\
\hline Crossover operator & single point \\
\hline Qty of seams & 8 \\
\hline Possible welding directions & 2 \\
\hline
\end{tabular}

\subsection{Convergence Analysis of Multi-objective GA}

We carried out a multi-objective GA experiment for seven generations and conducted the convergence analysis. Fig. 9, 1011 illustrate the behaviors of the four individuals (best, second best, worst and second worst) in terms of deformation, effective stress and multi-objective criteria as mentioned in equation 13. Elitism based selection method expedites the convergence of the GA. Figures show the monotonically decreasing values of the deformation over seven generations.

The best, second best, worst and second worst sequences are $(+6,-5,-2,+8,-1,+7$, $-3,-4),(-6,-5,-2,+7,-3,+8,-4,-1),(-4,-3,+8,-1,-6,-5,-2,+7)$ and $(-3,+8,-1,-6,-5,-2$, 
$+7,-4)$ respectively as can be seen in Figure 12 . Their maximum structural deformation and effective residual stress values are $0.65 \mathrm{~mm}$., $0.82 \mathrm{~mm}$., $2.0 \mathrm{~mm}$., and $2.08 \mathrm{~mm}$. and 427.68MPa , 424.40MPa , 425.20MPa and 425.96 MPa respectively as shown in Figures 13 and 14

To compute the minimum number of iterations necessary to ensure finding an optimal solution for GA with a prescribed probability $\alpha=0.98, \bar{m}=1, \beta=0.9$, number of bits required to represent an individual $l=8$ in equation 23 , we get $t \geq\lceil 6.95\rceil=$ 7. We conduct the multi-objective GA upto seven iterations since the computational complexity of the FEM based thermo-mechanical welding simulation approach is computationally very expensive.

Computation time. The average computation time for every individual of the GA using athermo-mechanical FEA approach using Simufact Welding $囚$ simulation software was 30 minutes. This time depends on the convergence of the thermal analysis.

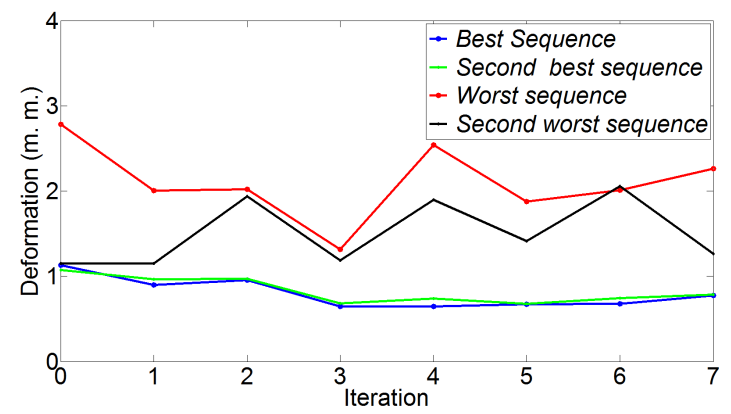

Fig. 9. Deformation.

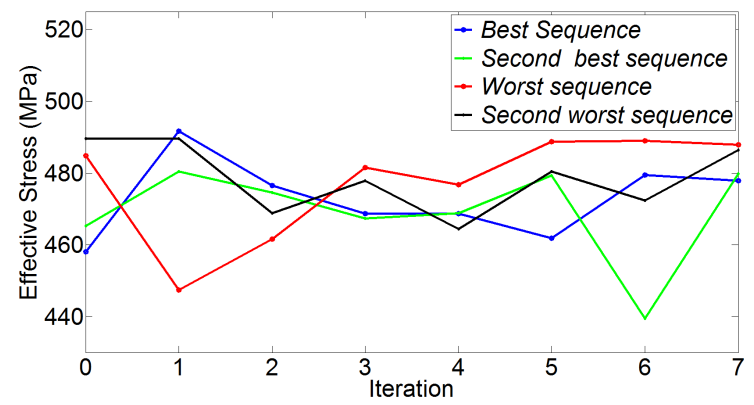

Fig. 10. Effective Stress. 


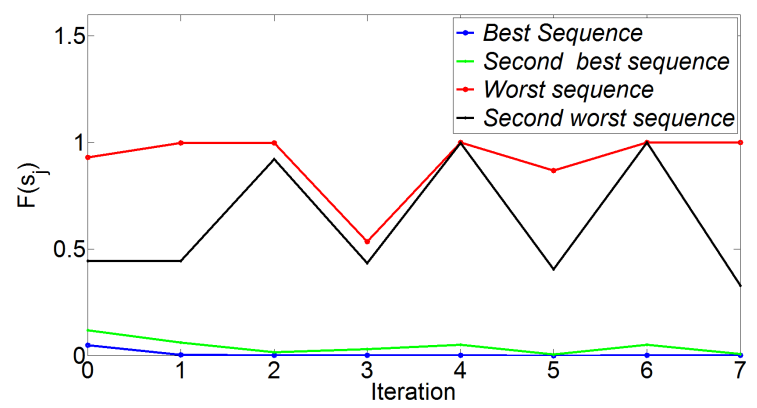

Fig. 11. Multi-Objective.

Figure 13 shows deformation distribution patterns for best, second best, second worst and worst sequences. It is clear that in worst and second worst sequences the maximum deformation value is greater than the best and second best sequences. Moreover, the distribution pattern is also better in the best ones, because the area near to the holes in the vertical pieces is less affected to pass a shaft or a bar through those holes.
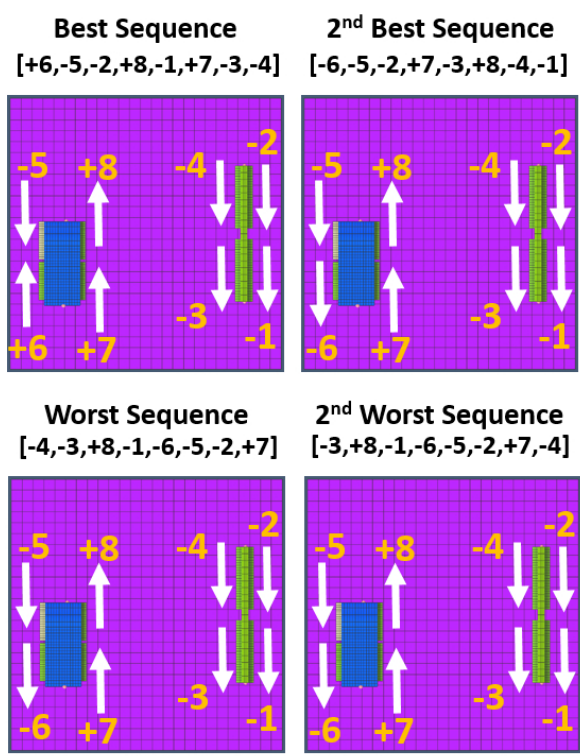

$2^{\text {nd }}$ Worst Sequence

$[-3,+8,-1,-6,-5,-2,+7,-4]$

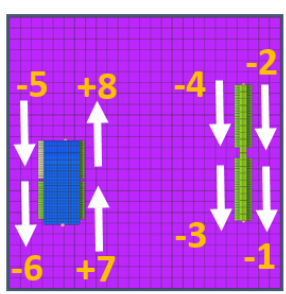

Fig. 12. Best, second best, worst and second worst sequences and their corresponding maximum structural deformation and effective stress.

Figure 14 shows effective residual stress distribution patterns for best, second best, second worst and worst sequences. The patterns are quite similar at the first looking, 
Deformation and Residual Stress Based Multi-Objective Genetic Algorithm for Welding ...

however there are differences in their frequency. The reduction in magnitude is lower than deformation. It is clear that effective residual stress affects the area close to the welding seam.
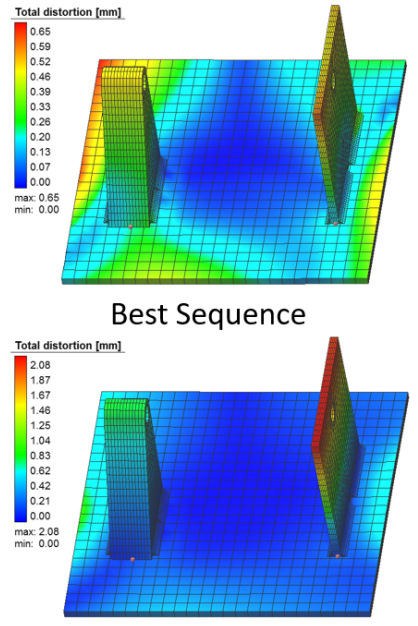

Second Worst Sequence

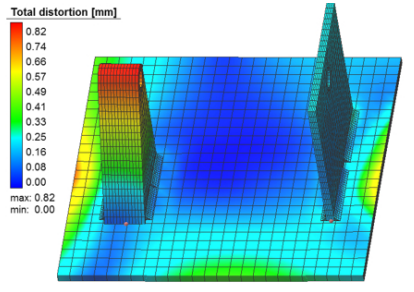

Second Best Sequence

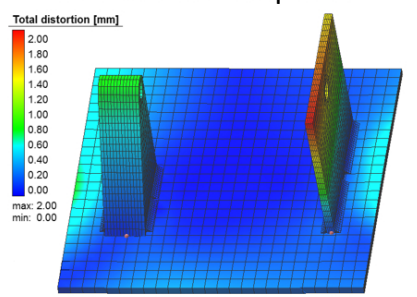

Worst Sequence

Fig. 13. Deformation patterns for best,second best, second worst and worst sequences.

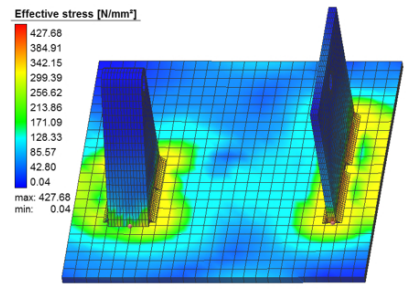

Best Sequence

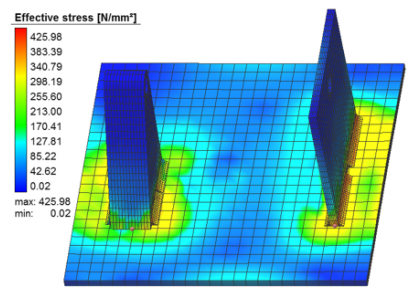

Second Worst Sequence

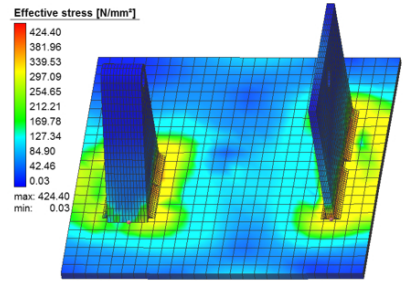

Second Best Sequence

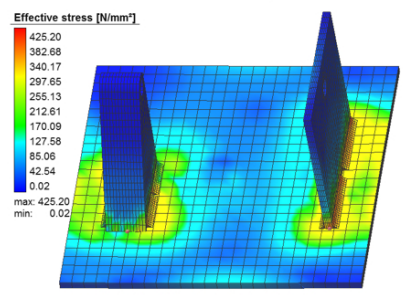

Worst Sequence

Fig. 14. Effective residual stress patterns for best,second best, second worst and worst sequences. 
Fig. 15 shows the values of the two terms, $w_{1} f_{1}$ and $w_{2} f_{2}$ of the multi-objective function over seven iterations of GA. This graph shows that the dynamic weighted method ensures an equitable treatment of each objective [7]. In this experiment, we always have $\left|w_{1} f_{1}-w_{2} f_{2}\right| \leq 0.07$ as shown in Fig. 15. Fig. 16 illustrates the Pareto front at iteration 1, 4 and 7 .

Results of the Pareto front shown in Fig. 16 demonstrate that both structural deformation and effective stress cannot be simultaneously reduced, decreasing the value of one increases the value of the other and vice versa. In this study, utilizing iterative dynamic weight selection based multi-objective GA, we find the optimal weight between deformation and effective residual stress through fair treatment of both of them.

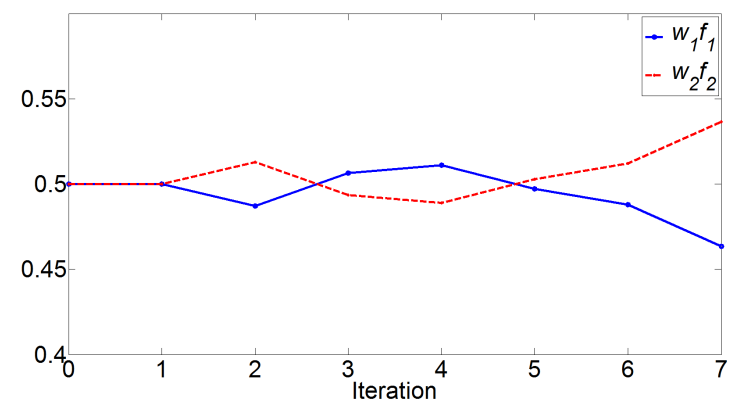

Fig. 15. $w_{1} f_{1}$ and $w_{2} f_{2}$ over dynamic weighted based multi-objective GA.

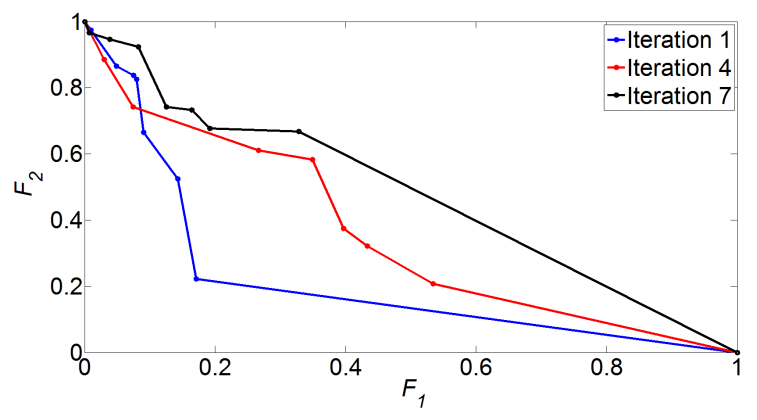

Fig. 16. Pareto Front.

\subsection{Effects of Welding Sequence on Welding Process Optimization}

Fig. 17, 18 and 19 demonstrate the normalized frequency of the deformation, effective residual stress and Multi-objective function (Equation 13) values of the best, second 


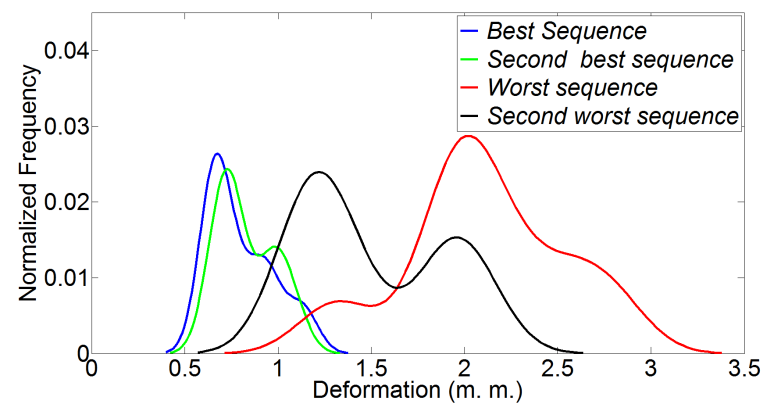

Fig. 17. Deformation.

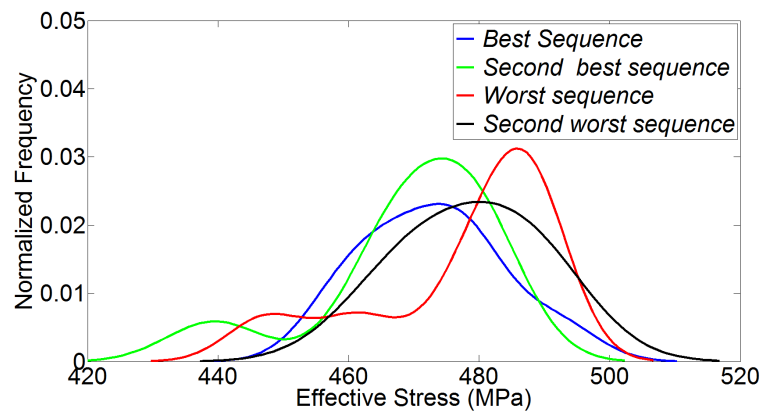

Fig. 18. Effective Stress.

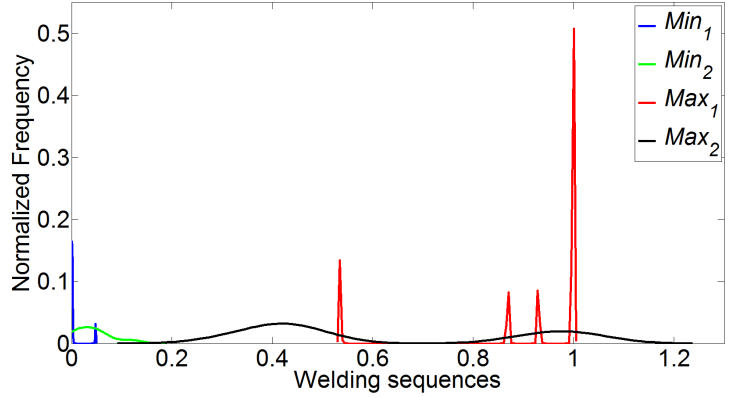

Fig. 19. Multi-Objective.

best, worst and second worst sequences respectively. Fig. 20, 21 and 22 demonstrate the deformation, effective residual stress and multi-objective values of these four sequences respectively in terms of the percentage if we consider the value of the worst sequence (red color bar) as 100\%. Fig. 20, 21, and 22 illustrate that best sequence (blue color bar) achieves $\sim 80 \%$ maximum structural deformation, $\sim 15 \%$ maximum effective stress and 
$\sim 60 \%$ maximum multi-objective values over worst sequence (red color bar) respectively. Fig. 20, 21, and 22 also demonstrate that both best and second best sequences obtains substantial reduction of maximum structural deformation, effective stress and multi-objective values over worst and second worst sequences (red and black bars are much taller than blue and green bars). This result clearly demonstrates that welding sequence has significant effect on welding optimization technique.

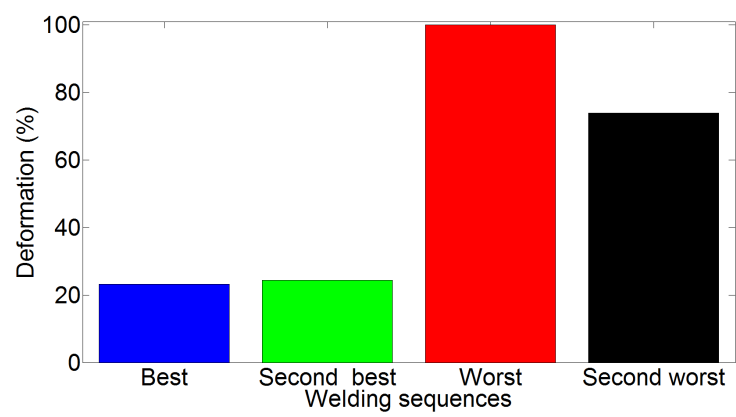

Fig. 20. Deformation.

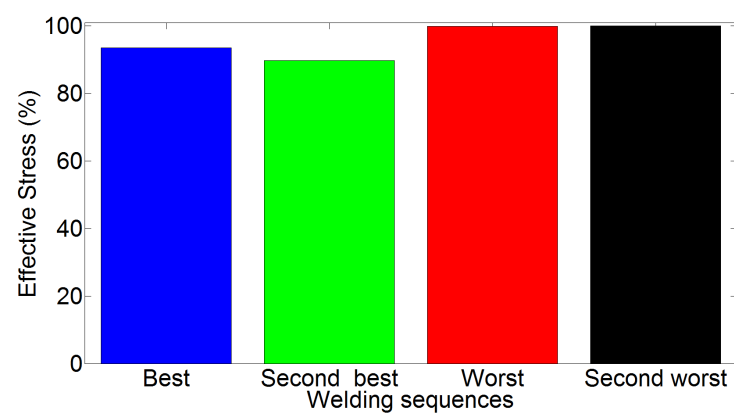

Fig. 21. Effective Stress.

\section{Conclusion and Future Work}

Structural deformation and effective residual stress defines a measurement of quality in terms of welded structures. In this research, we developed and implemented a multiobjective GA based on welding sequence optimization. Both structural deformation and effective residual stress have been combined. We exploited a multi-objective fitness 
Deformation and Residual Stress Based Multi-Objective Genetic Algorithm for Welding ...

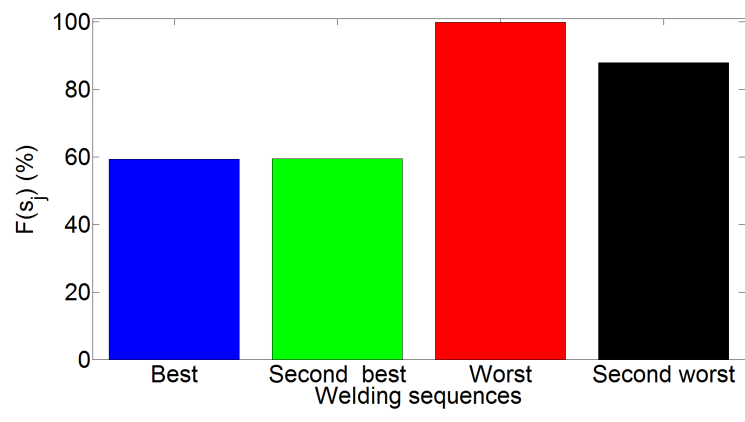

Fig. 22. Multi-Objective.

function which consists of the linear combination of the inverse of the structural deformation and effective residual stress have been linearly combined and the optimal weight between them are dynamically selected in an iterative manner ensuring an equitable treatment of both structural deformation and effective stress.

A thermo-mechanical FEA was used to compute the structural deformation and effective residual stress. The three sequential steps of GA: selection, crossover and mutation were tailored for welding sequence optimization to facilitate convergence and avoiding the repetition of the weld seam in the sequence.

An elitism selection approach was implemented by copying the three best individuals into the next generation to expedite the convergence as well as preserving the good chromosomes which have high probability to offer optimal solution. We implemented a sequential string searching algorithm to adjust the single point crossover algorithm for the welding sequence optimization to avoid the repetition of single bead into the welding sequence. For the similar reason, we only changed the direction of the welding seam instead the welding seam itself. We computed and executed minimum number of iterations necessary for finding the optimal solution of the GA based on the general Markov chain model of GA.

We carried out a simulation experiment on a mounting bracket which its design is widely used in vehicles and other applications. Experiments were conducted on a structure with eight weld seams. Results of Pareto front demonstrate that both structural deformation and effective residual stress cannot be simultaneously reduced. Experimental results illustrate that best welding sequence can reduce significant amount of structural deformation $(\sim 80 \%)$ and residual stress $(\sim 15 \%)$ over worst sequence.

This research launched multiple new directions to the welding sequence optimization. In future, we would like to incorporate other important characteristics to measure the quality of welded structures such as temperature, robot time and robot path for welding sequence optimization. Information about the structural deformation and effective residual stress after welding each seam in a sequence needs to be investigated. 


\section{References}

1. Alexandrescu, A., Agavriloaei, I.: Determining the best mutation probabilities of a genetic algorithm for mapping tasks. The Bulletin of The Polytechnic Institute from Iaşi LVII(LXI), $10(2011)$

2. Aytug, H., J., K.G.: Stopping criteria for finite length genetic algorithms. INFORMS Journal on Computing 8(2), 183-191 (1996)

3. Aytug, H., J., K.G.: New stopping criterion for genetic algorithms. European Journal of Operational Research 126(3), 662-674 (2000), http: //www. sciencedirect.com/science/ article/pii/S0377221799003215

4. Chapple, A., Tahir, Z., Jardine, F.: Weld Distortion Optimisation using HyperStudy. The 8th UK Altair Technology Conference 2013 pp. 1-13 (2013)

5. Derlukiewicz, D., Przybyek, G.: Chosen aspects of $\{$ FEM $\}$ strength analysis of telescopic jib mounted on mobile platform. Automation in Construction 17(3), 278-283 (2008), http://www.sciencedirect.com/science/article/pii/s0926580507000714, 8th International Conference on Computer-Aided Engineering

6. Fu, G., Loureno, M.I., Duan, M., Estefen, S.F.: Influence of the welding sequence on residual stress and distortion of fillet welded structures. Marine Structures 46, 30-55 (2016), http: //www.sciencedirect.com/science/article/pii/S0951833915001021

7. Gabli, M., Jaara, E.M., Mermri, E.B.: A genetic algorithm approach for an equitable treatment of objective functions in multi-objective optimization problems. International Journal of Computer Science 41(2) (2014)

8. Goldak, J., Asadi, M.: Challenges in verification of cwm software to compute residual stress and distortion in welds. In: ASME 2010 Pressure Vessels and Piping Conference: Volume 3. pp. 1-9 (2010)

9. Goldak, J.A., Akhlaghi, M.: Computational welding mechanics. Springer (2005)

10. Goldberg, D.E.: Genetic Algorithms in Search, Optimization and Machine Learning. Addison-Wesley Longman Publishing Co., Inc., Boston, MA, USA, 1st edn. (1989)

11. Hasançebi, O., Erbatur, F.: Evaluation of crossover techniques in genetic algorithm based optimum structural design. Computers \& Structures 78(13), 435-448 (2000), http: / www . sciencedirect.com/science/article/pii/s0045794900000894

12. Hussain, M.: Study of the dynamic behavior of a car body for mounting the rear axle. In: Proceedings of the European Automotive Congress (EAEC-ESFA). vol. 25 (2016)

13. Islam, M., Buijk, a., Rais-Rohani, M., Motoyama, K.: Simulation-based numerical optimization of arc welding process for reduced distortion in welded structures. Finite Elements in Analysis and Design 84, 54-64 (jul 2014), http://linkinghub.elsevier.com/ retrieve/pii/S0168874X14000201

14. Jackson, K., Darlington, R.: Advanced engineering methods for assessing welding distortion in aero-engine assemblies. IOP Conference Series: Materials Science and Engineering 26, 012018 (2011)

15. Kadivar, M.H., Jafarpur, K., Baradaran, G.H.: Optimizing welding sequence with genetic algorithm. Computational Mechanics 26(6), 514-519 (2000)

16. Kaya, Y., Uyar, M., Tekin, R.: A novel crossover operator for genetic algorithms: Ring crossover. CoRR abs/1105.0355 (2011), http://dblp.uni-trier.de/db/journals/ corr/corr1105.html\#abs-1105-0355

17. Kim, K.Y., Kim, D.W., Nnaji, B.O.: Robot arc welding task sequencing using genetic algorithms. IIE Transactions 34(10), 865-880 (2002)

18. Koenig, A.: A study of mutation methods for evolutionary algorithms. CS 447 - Advanced Topics in Artificial Intelligence pp. 1-8 (2002), http://web.mst.edu/\{ \}tauritzd/ courses/ec/fs2002/project/Koenig.pdf 
19. Kumar, D.A., Biswas, P., Mandal, N.R., Mahapatra, M.M.: A study on the effect of welding sequence in fabrication of large stiffened plate panels. Journal of Marine Science and Application 10(4), 429-436 (2011)

20. Liao, Y.G.: Optimal design of weld pattern in sheet metal assembly based on a genetic algorithm. International Journal of Advanced Manufacturing Technology 26(5-6), 512-516 (2005)

21. Lindgren, L.E.: Computational welding mechanics. In: Lindgren, L.E. (ed.) Computational Welding Mechanics, pp. 31-46. Woodhead Publishing Series in Welding and Other Joining Technologies, Woodhead Publishing (2007), http: / /www. sciencedirect.com/science/ article/pii/B9781845692216500045

22. Masubuchi, K.: Analysis of Welded Structures, vol. 3. Pergamon Press Ltd. (1980)

23. Mohammed, M.B., Sun, W., Hyde, T.H.: Welding sequence optimization of plasma arc for welded thin structures. In: WIT Transactions on The Built Environment. vol. 125, pp. 231242 (2012), http: / / library . witpress . com/viewpaper. asp?pcode=0P12-020-1

24. Soni, N., Kumar, T.: Study of various mutation operators in genetic algorithms. International Journal of Computer Science and Information Technologies 5, 4519-4521 (2014)

25. Studniarski, M.: Stopping criteria for genetic algorithms with application to multiobjective optimization. In: Schaefer, R., Cotta, C., Kołodziej, J., Rudolph, G. (eds.) Parallel Problem Solving from Nature, PPSN XI: 11th International Conference. pp. 697-706. Springer (2010)

26. Subbiah, S., Singh, O., Mohan, S.K., Jeyaraj, A.P.: Effect of muffler mounting bracket designs on durability. Engineering Failure Analysis 18(3), 1094-1107 (2011)

27. Xie, L.S., Hsieh, C.: Clamping and welding sequence optimization for minimizing cycle time and assembly deformation. International Journal of Materials \& Product Technology 17(5/6), 389-399 (2002) 